\title{
Seven years of recording from monkey cortex with a chronically implanted multiple microelectrode
}

\author{
Jürgen Krüger ${ }^{1,2}$, Fausto Caruana ${ }^{1}$, Riccardo Dalla Volta ${ }^{1}$ and Giacomo Rizzolatti ${ }^{1,3}$ \\ 1 Dipartimento di Neuroscienze, Università di Parma, Parma, Italy \\ 2 Bernstein Center for Computational Neuroscience, Hansastr. 9a, Freiburg, Germany \\ 3 Istituto Italiano di Tecnologia, Unità di Parma, Parma, Italy
}

\section{Edited by:}

Martin Stelzle, University of Tübingen, Germany

Reviewed by:

Silvestro Micera, Scuola Superiore Sant'Anna (SSSA), Italy

Gernot Mueller-Putz, Graz University of

Technology, Austria

*Correspondence:

Jürgen Krüger, Bernstein Center for

Computational Neuroscience,

Hansastr. 9a, D-79104, Freiburg,

Germany.

e-mail:kruger@brain.uni-freiburg.de
A brush of 64 microwires was chronically implanted in the ventral premotor cortex of a macaque monkey. Contrary to common approaches, the wires were inserted from the white matter side. This approach, by avoiding mechanical pressure on the dura and pia mater during penetration, disturbed only minimally the cortical recording site. With this approach isolated potentials and multiunit activity were recorded for more than 7 years in about one-third of electrodes. The indirect insertion method also provided an excellent stability within each recording session, and in some cases even allowed recording from the same neurons for several years. Histological examination of the implanted brain region shows only a very marginal damage to the recording area. Advantages and problems related to long-term recording are discussed.

Keywords: indirect insertion, multielectrode, chronic implant, monkey, premotor cortex

\section{INTRODUCTION}

The methods for getting access to cerebral activity range from recordings with microelectrodes and infrared spectroscopy to noninvasive techniques such as EEG, MEG, and fMRI. While all these methods have been mainly used for investigating brain functions, in recent years there is a growing interest in developing techniques to collect brain signals in order to help severely injured patients, or even, in a more futuristic view, to overcome some natural limits of normal brain function. Since MEG and fMRI have to be ruled out for technical reasons, the EEG appears to be the only noninvasive technique allowing one to extract signals from the brain of a freely moving human individual. As compared to its very rich signal structure, it is rather poorly suited for deriving reproducible information: the EEG signal allows to extract only less than 2 bits of information about a simple movement to reach a target; with sub-/ epidural electrodes a certain improvement can be obtained, which is however basically an invasive technique (Waldert et al., 2009).

When a patient presents severe clinical deficits, more invasive techniques appear to be justified in order to obtain clinical improvement or improvements in the quality of life. Indeed such techniques applied to animal models showed that neuronal signals can provide a rather reliable information for brain-machine interfaces. In a recent review Waldert et al. (2009) report that single and multiple unit signals provide the best informational yield, being also superior to local field potentials (LFPs). Of course, destructions of brain tissue have to be minimized, and only a single operation of implantation is desirable. Thus, chronically implanted microelectrodes or microwires appear to be the optimal procedure. An open question in this field concerns the stability of the signal during long-term recordings and, therefore, the development of pertinent reliable techniques. So far, the common knowledge of the notorious long-term instability of single unit recordings has been corroborated in a careful study by Tolias et al. (2007): using several tetrodes, they were able to re-identify the same individual neurons for several days up to several weeks so that an excellent basis for the study of intercellular learning processes was thereby demonstrated. However, beyond a few weeks the likelihood of recording from the same neurons became highly improbable.

Here we report our observations on single and multiunit recording carried out for more than 7 years after chronic implantation of 64 microwires into the ventral premotor cortex of a macaque monkey (Macaca nemestrina). We used a technique whose special feature is that the electrodes penetrate the cortex from the white matter side (see also Porada et al., 2000). In our study, the stress is less on long-term stability but on the mere fact that a chronic multielectrode can record single and multiunit signals for time spans that are relevant for clinical long-term implants.

\section{MATERIALS AND METHODS MANUFACTURE OF THE ELECTRODE}

Microelectrodes consisted of (initially) $100 \mathrm{~mm}$ long pieces of commercial $\mathrm{Ni}-\mathrm{Cr}-\mathrm{Al}$ wire (diameter $12.5 \mu \mathrm{m}$, polyimide insulation $3 \mu \mathrm{m}$ thick; "Isaohm", Isabellenhütte Dillenburg, Germany). One end of each wire was de-insulated at a length of $1 \mathrm{~mm}$ by mechanical scratching. In a block of $8 \times 8$ female plugs (inter-contact distances of about $1 \mathrm{~mm}$ ) each plug was contacted from behind to the deinsulated end of a wire by silver-epoxy "Epo-Tek" H20E (Polytec, 76337 Waldbronn, Germany) (Figure 1A).

The space around these contacts was then carefully filled up with household epoxy (“Uhu-endfest 300”, 77815 Bühl, Germany). This detail is of importance since we had observed earlier that moisture might reach the plugs from underneath once the plug is fixed to the animal skull by acrylic cement.

The contacted wires were glued together to form a bundle by a solution of plexiglas in dichloroethane which allows a redissolution in case of mistakes. It is important to take care to 
A

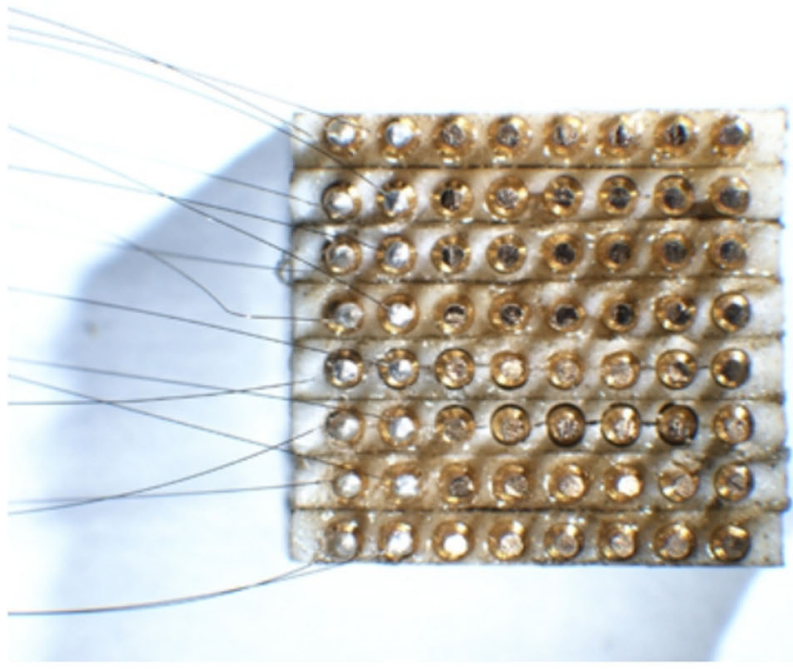

B

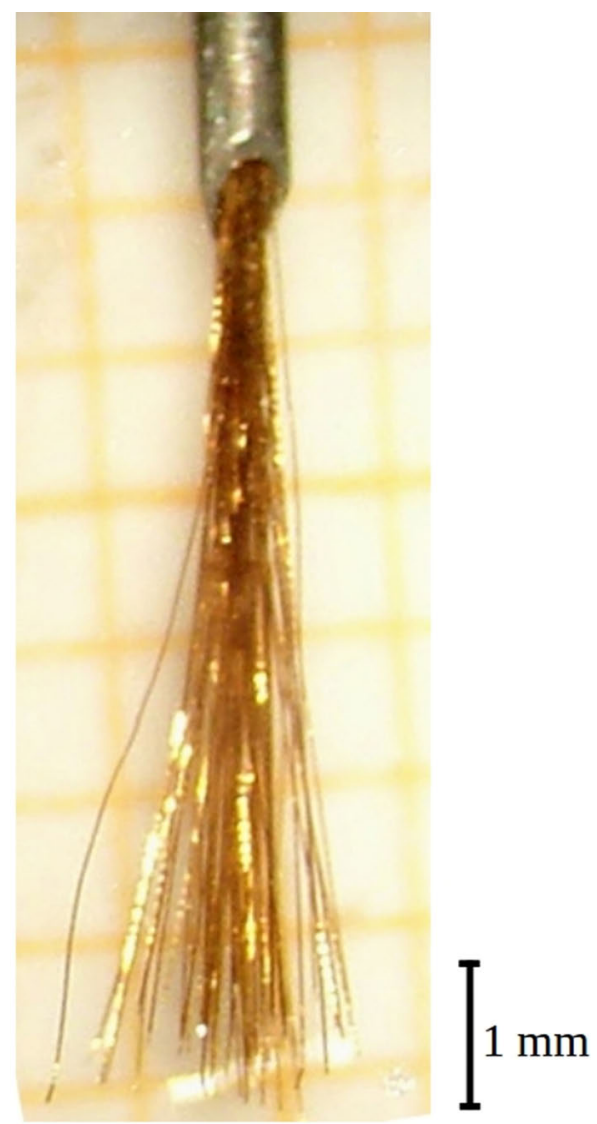

FIGURE 1 | Electrode construction. (A) View of the 64-fold plug from underneath while 16 wires have already been contacted by silver epoxy (leftmost two columns). After curing they will be covered by insulating epoxy and then the next two columns be contacted. The distance between two contacts is $0.9 \mathrm{~mm}$. (B) View of a ready 64-fold electrode brush protruding from the cannula (top).

keep the wires straight. The glued bundle is then less than $0.3 \mathrm{~mm}$ thick. The bundle is then introduced into a syringe cannula (outer diameter $0.6 \mathrm{~mm}$ ) bent to the shape of a circle sector (curvature radius $13 \mathrm{~mm}$ ). Thereafter it is cut to equal length of all wires. A small drop of sugar, molten by a heated hairpin wire, is applied at $6 \mathrm{~mm}$ far from the bundle end, and the plexiglas glue is dissolved from the wire tips up to that barrier. Individual wires are then shortened by up to $1 \mathrm{~mm}$ so that a brush with a $1 \mathrm{~mm}$ wide spectrum of lengths is obtained (Figure 1B). The electrode tips are just formed by the bluntly, sometimes obliquely cut wire ends.

As a test, the electrodes, forming a brush about $6 \mathrm{~mm}$ long, were immersed in water. In order to identify each electrode, $-5 \mathrm{~V}$ DC was applied to each plug contact in turn while the appearance of a bubble was observed under a microscope. This was done for two orthogonal inspection angles so that a spatial drawing of the locations of the properly numbered electrode tips was obtained. Of course in lateral directions that distribution might change when the electrodes penetrate the brain, while the knowledge of the individual lengths remains valid.

\section{IMPLANTATION}

An adult female macaque monkey (M. nemestrina) weighing $5 \mathrm{~kg}$ was used. All experimental protocols were approved by the Veterinarian Animal Care and Use Committee of the University of Parma and complied with the European law on the humane care and use of laboratory animals. Some months before the implantation two small titanium screws (lengths $3 \mathrm{~mm}$, thread diameter $0.9 \mathrm{~mm}$; Medizinund Dentaltechnik, 78052 VS-Villingen, Germany) were inserted in the skull of the animal under ketamine anesthesia, near the probabilistically estimated target locus in the ventral premotor cortex. A magnetic resonance image of the head was then taken in which the exact target area and the screws were visible (Figure 2A).

Several weeks later the monkey was operated under general anesthesia (ketamine hydrochloride, $15 \mathrm{mg} / \mathrm{kg}$ i.m.). The heart rate and expired $\mathrm{CO}_{2}$ were continuously monitored. The skin was opened on the skull, and a bone trepanation $(\varnothing 8 \mathrm{~mm})$ was made about $20 \mathrm{~mm}$ caudally from the target site which remained covered. Five titanium screws (lengths $6 \mathrm{~mm}$, thread diameter $1.5 \mathrm{~mm}$; type \#400.006, Synthes, 79224 Umkirch, Germany) were partly lowered into the skull at spatially distributed loci. One of them was connected to a ground contact.

The implantation procedure is depicted schematically in Figure 2. Its essential feature is that the cortical target locus is reached from the white matter side although in principle it would also be accessible from the surface. No pia mater has to be penetrated, and less blood vessels are encountered as compared to a surface approach. A special carrier carrying the cannula on a circular path, and a "skull pointer", are the principal elements. 
A

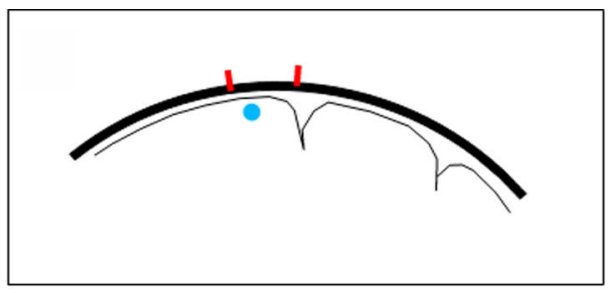

B

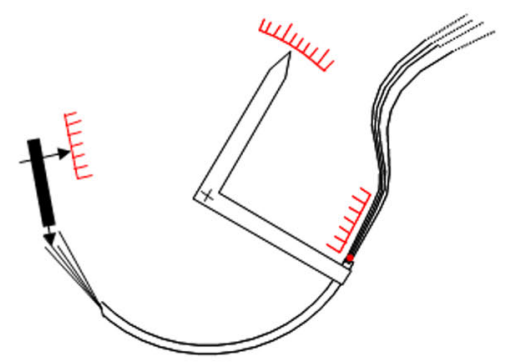

C

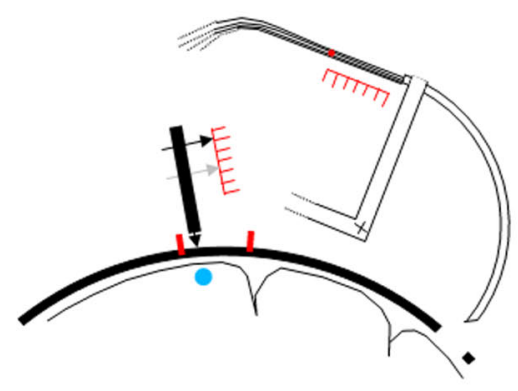

D

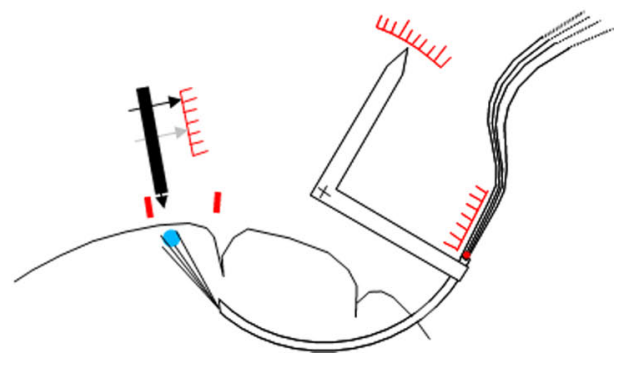

FIGURE 2 | Schematic procedure of the implantation. (A) Schematic drawing of a magnetic resonance image showing the brain to be investigated, with the target area (blue point) and two small titanium screws (red) in the nearby bone. (B) Adjustment of the device before the implantation. A "skull pointer" (left black arrow) is lowered into the middle of the region of the electrode tips, and its position is noted on a scale. The angular position of the rotating device carrying the cannula (white arrow), and the extent of insertion of the bundle of wires into the hind end of the cannula, are also noted on corresponding scales. (C) The skull pointer is retracted by the bone surface-target point distance, the cannula with the electrodes is rotated away, and the electrodes are retracted into the cannula. Guided by the positions of the screws the pointer tip is then brought in contact with the animal's skull, and a far lateral trepanation (right) is made. (D) The cannula is rotated into the brain, and then the bundle of wires is pushed forward, until the two previously noted positions are reached. An additional feature of the device (not shown in the figure) is that the entire system of pointer and cannula carrier can be rotated around the selected target point. In the case of a disturbing blood vessel encountered at the intended entry point within the trepanation, another entry site can easily be selected without new adjustments.
In a first step (Figure 2B), the system was calibrated by taking several scale readings when the brush of electrodes reached a given target point, defined by the skull pointer. The electrode brush was then retracted into the curved cannula, and the cannula and its carrier were rotated away (Figure 2C). The skull pointer was then pulled back by the distance between the skull surface and the target point, as determined by the magnetic resonance image. Thereafter the animal was brought into contact with the skull pointer in this position. The cannula was inserted into the brain on a circular path through the previously opened hole and an opening in the dura until the predetermined scale reading was reached. Thereafter the electrode bundle alone was pushed until the corresponding scale reading was attained (Figure 2D).

The cannula and the bundle within the cannula were then fixed to the bone first by blue-light curing cement ("Durafill Bond"; Heraeus Kulzer, 61273 Wehrheim/Ts, Germany), and then by acrylic cement ("Technovit 5071"; Heraeus Kulzer, 61273 Wehrheim/Ts, Germany) which was anchored around the bone screws, and which also held the plug, the ground contact, and some nuts to hold later the preamplifier and a cover. The block of cement measured about $4 \times 5 \mathrm{~cm}$ around which the skin was sutured.

\section{DATA RECORDING}

During the recordings the animal sat in a restraining chair. She could freely move her head. A small non-commercial 64-channel preamplifier ("headstage") was mounted on the head. The signals were then amplified and recorded with a sampling rate of $19 \mathrm{kHz} /$ channel by a PC. An online multi-channel oscilloscope-like program allowed us to inspect the raw signals. A typical experiment lasted from 6 to 60 min with the data being continuously recorded from all channels, and then stored on a hard disk. The signals were frequency limited by hardware filters between 5 and $19000 \mathrm{~Hz}$, and to see the action potentials ("spikes") a $500 \mathrm{~Hz}$ high-pass was applied by a software.

\section{DATA ANALYSIS}

The stored data were inspected offline with another multi-channel oscilloscope-like program which also allowed the spikes to be triggered by setting amplitude levels by eye. Spike sizes of up to three levels could be triggered separately from each single channel, when the visible spikes had a sufficient magnitude, and when families of smaller and larger spikes could be distinguished by eye. Triggered events at lower levels were eliminated if an event at a higher level had been found.

The segregation done in this way is imperfect insofar as there is no proof that the signals extracted at a given trigger level stem from one or from several cells. However, since we were more interested in multineuronal excitation patterns, the stability of that segregation along the duration of the experiment was more important, and indeed one rarely observed changes of spike sizes during a single experimental session. For further details about the usefulness of this type of multineuronal signal see Discussion.

\section{Intact recording channels recording no spike versus technically defective channels}

At the outset of the present series of experiments we were interested in recording useful signals from a sufficiently large number of electrodes but we did not take care of the difference between a nonfunctioning recording channel and an intact channel recording no 
spike. However, in order to give a relevant picture of the recording quality, we had to split these two cases, all the more so as sometimes the fraction of technically non-functioning channels was very high. Therefore, in the stored data we had to identify the truly functioning electrodes, and relate the determined recording qualities only to them.

The main technical defect was a lack of contact, due to impurities, between the plug on the animal's head and the headstage input. It turned out that an interruption at that level resulted in an about twofold widening of the baseline noise, as compared to a normally functioning electrode. We frequently encountered cases where due to movements of the animal the contact opened and closed during a recording, yielding oscilloscope traces such as shown in Figure 3.

We also could increase the number of contacted channels by forcing the block of male plugs on the headstage further into the female plugs on the monkey's head.

The signals from all correctly operating electrodes had very much the same noise level (width about $50 \mu \mathrm{V}$ ) to the extent that a single fixed trigger level for all electrodes would have yielded fairly acceptable results except, of course, for the multilevel triggering. This feature allowed us to identify the channels whose contacts were interrupted at the input level of the preamplifier, by their increased width of the noise band. In some experiments we had accepted rather high proportions of interruptions of this type because otherwise the animal would have had to be anesthetized in order to clean the contacts. Other technical defects occurred only in small minorities of channels, and were easily recognizable without doubt.

\section{HISTOLOGY AND ELECTRODE REEXAMINATION}

The monkey was anesthetized with ketamine hydrochloride (15 mg/kg, i.m.), followed by an i.v. lethal injection of pentobarbital sodium, and perfused through the left ventricle with warm buffered saline followed by fixative (paraformaldehyde 3.5\%).

The skull part to which the curved cannula was cemented was carefully removed from the perfused brain. The brain was then removed from the skull, photographed, and then frozen, cut coronally (section thickness $60 \mu \mathrm{m}$ ) and Nissl-stained.

The electrode brush, still in contact with the block of plugs, was reexamined by the water test in the same way as before the implantation (see above). The spatial tip distributions before and after the experiment only showed a rather coarse quadrant-to-quadrant correspondence within the brush while closer neighborhood relationships were not conserved. We conclude that also the unobservable distribution within the brain could not be deduced with a higher accuracy from the spatial pre- and post-implantation distributions, except, of course, for the lengths of the individual electrodes.

At the occasion of the final electrode re-examination we noted that a few electrodes showed broken insulations so that the bubble not only showed up at the electrode tip but in addition at a point remote from the tip. However, we could not find a systematic hint that these points had allowed a supplementary recording of neuronal activity.

\section{RESULTS}

The electrodes were implanted on December 3, 2001. Data from 75 recording sessions were used, distributed over 2729 days corresponding to nearly 7.5 years. Data from each session were triggered by eye at up to three levels as described in Section "Data analysis". Most technically non-functioning recording channels were identified by the criterion explained in Figure 3, and in a few cases by other straightforward criteria (e.g. broken wires). An overview of the results is given in Figure 4.

We have distinguished electrodes on which one, two, or three spike magnitude levels could be discriminated (rectangles of different heights). In addition, there are electrodes that show no spike activity although technically they are working properly (blanks). Dots indicate non-functioning electrodes or channels.

It can already be seen in Figure 4, irrespective of the distribution of non-functioning channels, that a continuous degradation of the recording quality with time is not obvious. It would be recognizable by an overall decrease of heights of the rectangles in the lower parts of the figure. The figure also shows that the recordings are not stable from one day to the next. However, it is not very helpful to define a statistically defined probability of "rectangle change" as a function of time because the aspect of rectangle height alone overestimates the stability since the mere appearance of a rectangle of a given height does not indicate that activity of the same neurons has been recorded.

It may also be noted that the changes among days do not follow the hypothesis of sudden changes on many electrodes, and periods of stability in between, in agreement with the observations of Porada et al. (2000). Such a behavior would be expected if gross accelerations of the tissue against the electrodes were a major factor of instability.

The true overall recording quality has to be judged relative to the number of technically intact electrodes or channels. Therefore we have determined, for each session, the number of intact channels. This number is shown as a dashed curve in Figure 5.

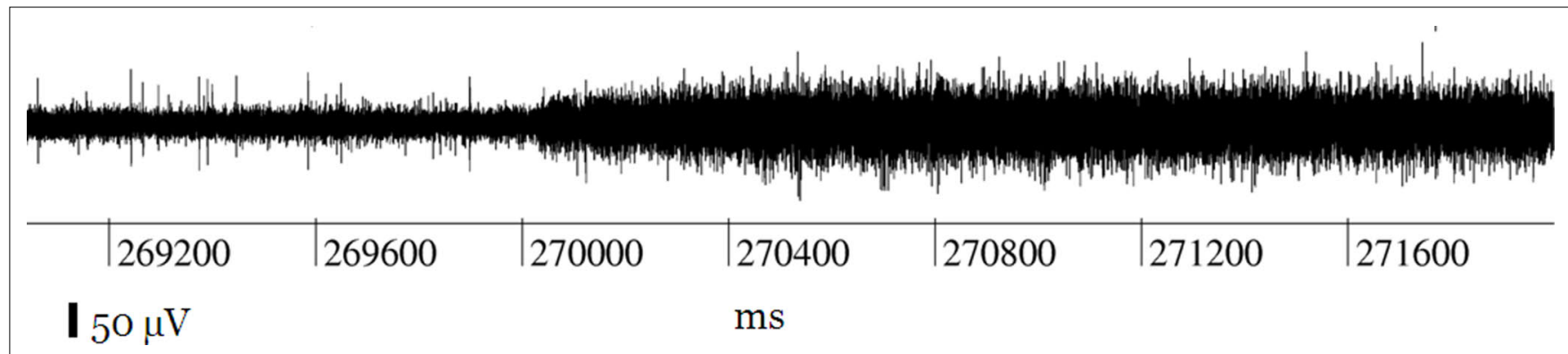

FIGURE 3 | Record of a channel showing the typical effect of an interruption of the contact within the plug on the head of the animal: the spikes disappear, and the width of the noise level is duplicated. 
Instead of expressing the numbers of channels with 1, 2, 3, or 0 spike trains as a fraction of the total of 64 electrodes, these numbers were related to the number of intact channels, that is, to the height of the dashed curve. This is equivalent to a normalization to fictive 64 intact channels. For each recording day, these relative counts are displayed along the time axis in four curves. In order to avoid crowding of points, in some sections

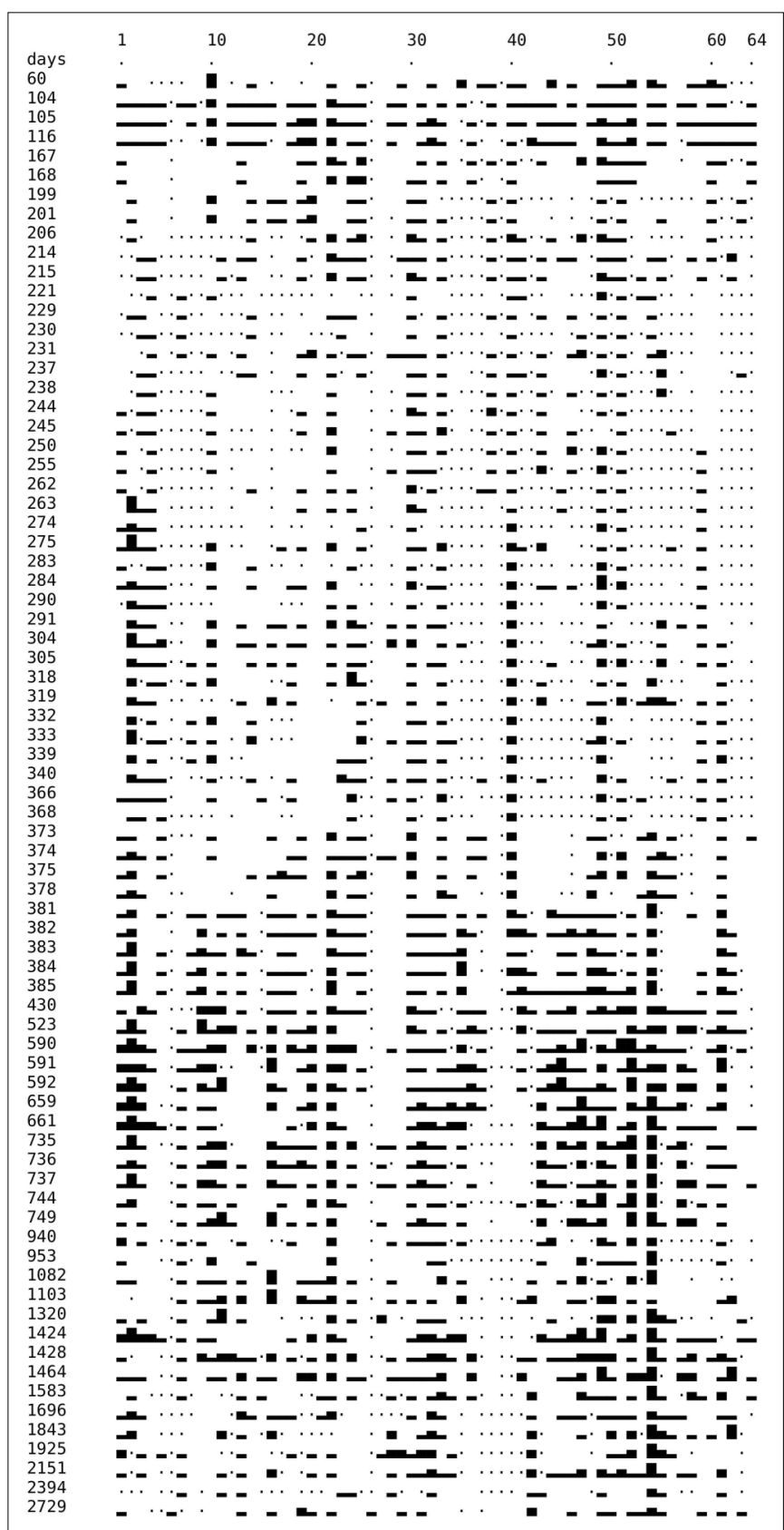

FIGURE 4 |Temporal development of recording quality. Left column: time (in days) after implantation. Symbols: Dot: technically defect electrode or amplification channel. Blank: technically intact, but no spike recorded. Rectangles of different height: 1,2, or 3 spike trains recorded, respectively, by each one electrode. On electrode no 2 on days 263 and 275 we could properly discriminate four different spike trains. This is rendered by higher rectangles but the category "four spike separable trains" was not considered henceforth. where larger numbers of experiments had been done in a few days, the individual channel counts have been averaged over up to six successive days.

It can be seen that the number of intact channels goes down around day 275 to only 31 channels still operating properly. Before day 373 , that is, about a year after the implantation, a substantial technical revision was executed; in particular, the female plugs on the head of the animal were cleaned under anesthesia, and the cover of the plugs was improved. This lead to a considerable increase of the number of technically intact channels which from that time on oscillated around 50 .

Figure 5 shows that essentially the number of channels showing no spike activity (gray) was all over the 7 years grossly around half of all working electrodes, with notable exceptions around 100 and 500 post-operative days where we observed clear neuronal activity on more than two-thirds of the electrodes. Most changes seen in the continuous curves were not related to any known event.

After about 1 year we noted an increase of the number of channels showing more than one isolated spike. However, in many of these cases this does not mean that two or three isolated spikes appeared on more and more channels, but rather that there was an increasing spectrum of spike sizes for which it was possible to segregate the activity in several trigger levels that indeed often showed different activity patterns in relation to the pertinent experimental conditions. It was noteworthy that this evolution was not accompanied by an overall decrease of spike sizes, as one would imagine

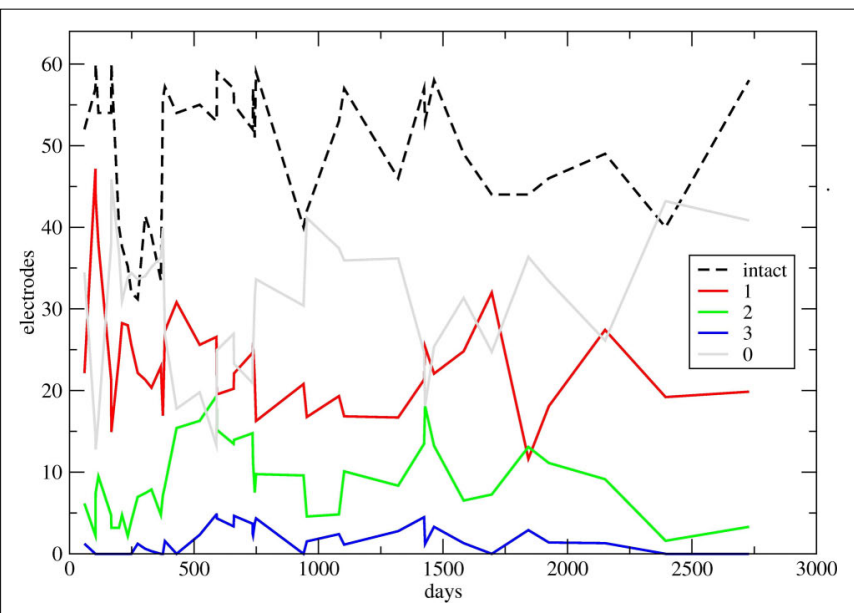

FIGURE 5 |Temporal development of recording quality, summated over all electrodes. In some sections of the diagrams, many experiments done within a few days led to a crowding of symbols; in these cases up to six successive counts have been averaged, without loss of the general character of the curves. Black dashed curve: for each day (or up to 6 days), number of technically intact electrodes or channels. Continuous curves: for each day (or up to 6 days), number of electrodes recording 1,2,3, and 0 spike trains (red, green, blue, and gray), respectively, normalized to 64 intact electrodes. For the normalization, for each day the corresponding numbers have been multiplied by 64 and divided by the height of the dashed curve. The higher value of technically intact electrodes on the last day is due to a previous cleaning of the plugs, and in addition to a threefold permutation of electrodes and headstage/ amplificator channels so that the true recording quality of many more electrodes could be examined. 
when nearby cells die, and a subsequent filling in of the space by inactive tissue takes place. In this case one would have expected a clear-cut general deterioration of the signal to noise ratio which was not observed actually. We only noted a slight overall decrease in spike size on all channels several years later; it is in a representative way reflected by the diminishing sizes of the spikes in Figure 6A.

Several obvious cases of spikes trains of the same neuron recorded by two or more electrodes (Figure 6B) were only observed in the first year. However, we did not attempt to determine cases of more subtle multiple recordings by correlation analyses.

A peculiar case was channel 54 on which we often recorded the largest spikes out of all channels (Figure 6A). The astonishing feature was that, beginning about 1 year after the implantation, there were always two trains of very large spikes until the end of the recording period 7 years later. In contrast, there was no channel on which one spike train was observed continuously over such a long-time span. The mere improbability of two trains of large spikes, both showing always a high firing rate, makes it very likely that we recorded from the same two neurons for 7 years. Functional neuronal properties determined in two experimental series 4 years apart supported this contention. For the remaining electrodes, one could not be confident, from one recording to the next, even when separated by only one day, that the recording was obtained from the same neuron.

A case in point for purposes of long-term recording was our observation, in experiments where the same set of visual scenes and motor tasks were applied for many days in succession, that the motor-related activity remained fairly clear-cut (although dissimilar from one day to the next) while there was an overall decrease of the visual responses. It would have been possible to draw false conclusions about the state of the target area if only the visual responses had been recorded. On the other hand, this observation points to a methodological possibility in the case of temporally unstable recordings (see Discussion): if the activities are unstable from one day to the next, one has to focus attention on the temporal evolution of similarities and dissimilarities among activities observed in the same recording session.

The plugs were cleaned just before the final recording session (right end of the curves in Figure 5). In that recording we also have commuted recording channels in several ways, in order to circumvent all sources of technical defects so that the number of technically intact channels rose to 58. Spikes were observed on 21 electrodes, with a few of them allowing a separation into two size classes. The overall spike size had somewhat decreased in that recording, and also the one taken a year earlier. However, already in the first years of recording we had noted such periods of decreasing spike sizes that were followed later by sudden increases. We have interpreted these changes as resulting either from processes related to skull growth or other sources of displacement of the bundle of electrodes against the nervous tissue.

The inspection of the Nissl-stained histological sections showed the curved trajectory of the cannula passing through the white matter below the agranular frontal areas. The electrode tracks entered the gray matter of the ventral premotor cortex from the deep layers at the level of the crown of the posterior bank of inferior arcuate sulcus. Around the hole left by the root of the electrode brush moderate signs of gliosis were visible, mainly at the border between layer VI and white matter. In the cortical portion where the electrodes separated apart in the gray matter, some spots of gliosis were visible; often they were close to blood vessels that had probably been pierced. In the most relevant area, namely the middle of the "cloud" of electrode tips we found a surprisingly intact tissue (Figure 7).

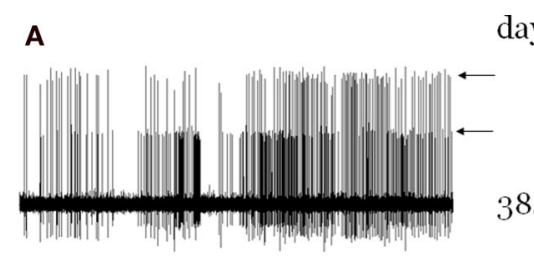

day

B
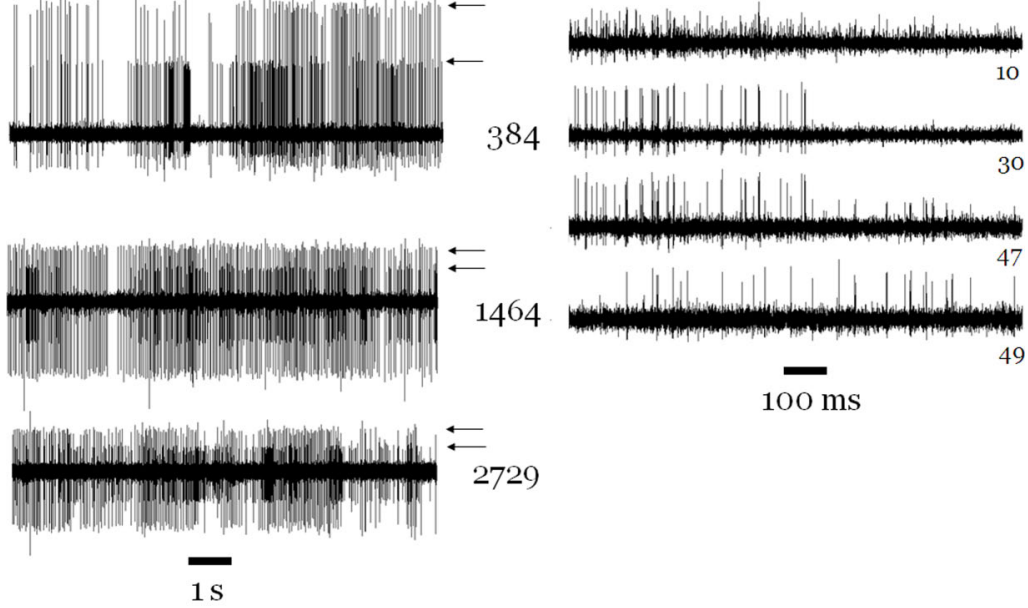

FIGURE 6 | (A) Sections of recordings from channel 54 separated by more than 6 years, showing two trains of spikes (arrows). From day 384 on, in all recordings with intact channel 54, two trains of well-isolated spikes, with high ongoing discharge rates, were seen. On a few days (not shown) a smaller third spike train appeared. The noise level width is about $50 \mu \mathrm{V}$ in all cases. (B) Four simultaneous recordings on day 168. The spike trains on channel 30 (number at right) can be seen also to appear on channels 10 and 47. Another train of smaller spikes on channel 47 can be seen also to appear on channel 49 (right side). 
It was noteworthy that the visible gliosis was not diffuse, but spotty and localized (four upper arrows). The lower arrow points to a gliosis which was more extended in adjacent sections further towards the root of the electrode brush (not shown), and which was adjacent to a blood vessel filled with detritus. To sum up, the anatomy of this region was not subverted, the division in layers was evident, the pyramidal neurons were visible, normally packed and the cytoarchitectonic organization was normal.

\section{DISCUSSION}

Here we have shown that an inverted insertion of microelectrodes into gray matter can improve the quality of the neural signal, allowing to record from primate cortex for more than 7 years. Many years after the implantation neuronal signals were detected in experiments during which cellular activity continued to be elicited by means of tests developed in our laboratory (Umiltà et al., 2008).

We stress that the purpose of our study is not to demonstrate that one can record from the same neurons for time spans that are relevant for a chronical access to the nervous system. Nor do we intend to demonstrate that different but always well-isolated single units can easily be recorded for such time spans (although this may be feasible with advanced spike-sorting methods such as the one applied by Tolias et al.2007). Rather, it has to be borne in mind that

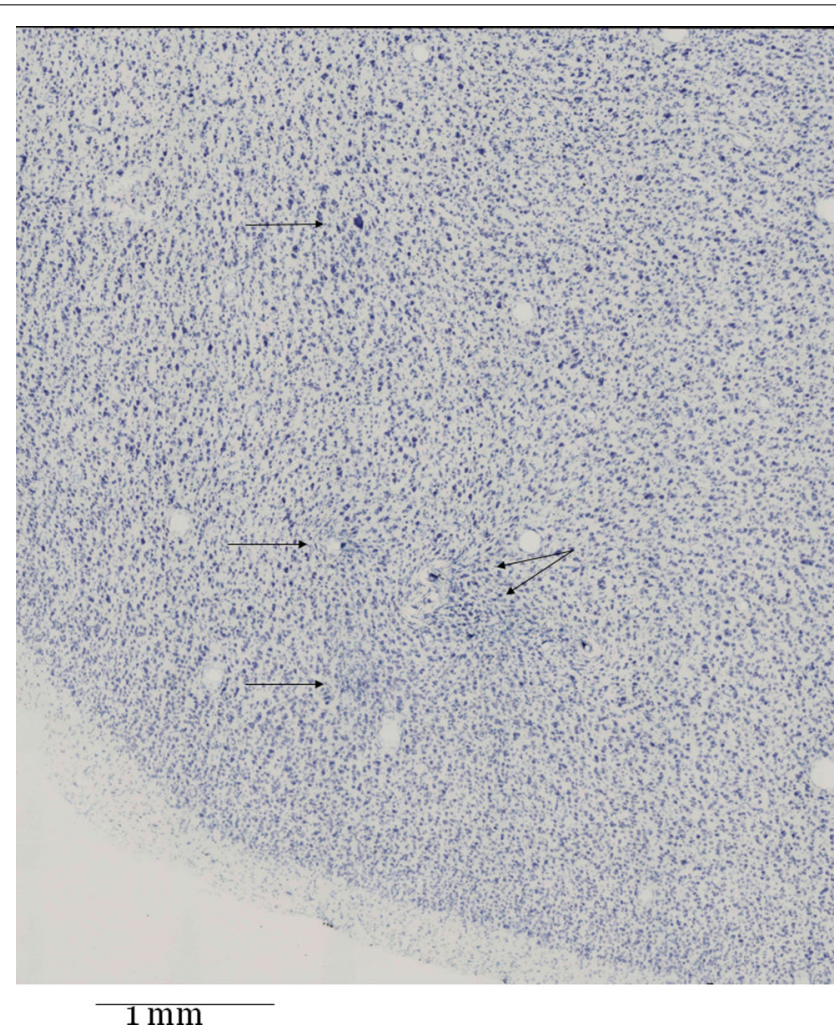

FIGURE 7 | Nissl-stained frontal section (thickness $60 \mu \mathrm{m}$ ) through the middle of the "cloud" of electrode tips. The location is the F5 region in the posterior bank of the arcuate sulcus just lateral/below its spur. The four upper arrows point to localized gliosis. The lower arrow points to a lesion near a larger blood vessel filled with detritus. The lesion is more prominent in adjacent sections (not shown) towards the root of the brush of electrodes. any useful long-term recording of neural activity from the human brain will be a true multineuronal affair to which multineuronal signals from each electrode can very well contribute, and which is not simply the sum of known activity characteristics of a number of single neurons.

Despite the described limitations arising in long lasting implants, it has been shown that multineuronal activity is optimal for decoding an excitation pattern (see the reviews of Donoghue, 2008, and Hatsopoulos and Donoghue, 2009). Yet, LFPs are successful as well (Mehring et al., 2003; Andersen et al., 2004): they promise a better long-term stability, and may be advantageous when several parameters have to be decoded. Multineuronal signals have been successfully employed to control an artificial motor device (Laubach et al., 2000; Serruya et al., 2002; Hochberg et al., 2006; Stark and Abeles, 2007; Velliste et al., 2008; Fraser et al., 2009). For this reason, a scientific advance would be obtained by matching long-term stability, at present better offered by LFPs, with the decoding quality offered by multineuronal activity. Here we have shown that the adoption of indirect insertion allows to record multineuronal activity for a long time.

Although being optimal with respect to informational richness one has to accept that single or multineuronal signals will not be the same from one day to the next. Under this condition it makes no sense to focus attention on the activities of well-isolated single neurons. Indeed Stark and Abeles (2007), and Fraser et al. (2009) were successful with techniques that may be compared to our spike extraction method.

Thus, how can one cope with instability? Since the recording of long-term multineuronal activity was rather an exception, little is known about the effects of long-term instability on the decoding performance of multineuronal excitation patterns. Consider a relationship having been established on a given day between an experimental condition and a neuronal pattern. In our recordings we could not be confident that this relationship remained valid for several days or weeks, because the excitation patterns might change due to instability. Yet, if the relationship has few defining parameters, as in a map (e.g. receptive field positions; Jain et al., 2001) even an unstable recording may yield rather stable results over longer time spans, due to the similarity of receptive field locations of neighboring neurons.

A general solution for the long-term instability problem may be that relationships between neuronal patterns, established on the same day, remain more stable over time, even if the patterns themselves undergo substantial changes. A promising method for visualizing relationships between multineuronal excitation patterns is Multidimensional Scaling (Dalla Volta et al., 2002). A case in point is the observation, within a single recording session, how the pattern visually evoked by an unknown artificial grasping instrument becomes comparable to the one evoked by a grasping hand, due to a learning process (work in progress). It is expectable that the initial dissimilarity changing into a similarity is not limited to just the ensemble of neurons being recorded. If this were true, it would be extremely unlikely to find such a relationship. Rather, the patterns of other nearby neurons must also show this relationship, although the patterns themselves will be dissimilar. Thus, a desired decoding, or any other scientific conclusion, would have to be based on such quasi-simultaneous intracerebral relationships. Observations of this type will be important for the follow-up of non-repeatable 
processes such as learning, or the effects of diseases or therapeutic measures. They cannot be obtained with conventional single cell recording technique.

The ultimate consequence along these lines would be to find a transformation rule describing the multineuronal change from one day to the next: a patient depending on a multimicrowire implant would each day have to make a test which determines the changed state of affairs. If it could be proven that a transformation rule can be found that is valid for many more conditions than the ones covered by the test, the instability question would have a solution.

Now some points related to anatomical changes will be discussed. Spikes were observed at two-thirds of all electrodes mainly during the first year, after which many cases of spike trains overtly recorded by more than one electrode disappeared. This observation is probably due to a loss of neurons in immediate vicinity of the electrode tips occurring within the first months after the implantation. It is possible that the neural tissue retracted from the vicinity of the electrodes, thereby decreasing the number of spike trains available for single-unit isolation. However, as far as we could judge, the functional properties of the cerebral area remained unchanged. There was the additional fact that the spike sizes did not appreciably decrease when, after some months, the signals of more and more channels tended to originate from more than one neuron. It reminded the effect of an increase of the tip surface of the electrode, but we ignore its exact nature.

Similar mechanisms certainly operate when an array of microelectrodes ("Utah" device; Nordhausen et al., 1996) is introduced into the cortex from the surface. Szarowski et al. (2003) have noted limitations for long-term applications of this type of device. The additional feature of many more blood vessels being located at the surface than in the white matter will increase the reported adverse effect of destroyed vessels so that after some months gliosis was observed to fill the entire space between the electrodes (Rousche and Normann, 1998).

Classical surface approaches are concerned by gliosis related to the speed of introduction of the electrodes into the brain tissue. The "Utah" device seems to suffer from the fact that it is usually advanced very rapidly in order to profit from inertia of the tissue when penetrating the dura mater. Yet, the recording properties of the device are excellent for shorter times (Nordhausen et al., 1996). Indeed, Nicolelis et al. (2003) recommend a slow speed of tissue penetration, when using multiple wires. However, the authors do not mention the severe gliosis effects, as reported by Rousche and Normann (1998). Yamamoto and Wilson (2008) recommend an extremely slow penetration speed of $2-5 \mathrm{~m} \mu / \mathrm{s}$, limited to no more than 50-100 $\mathrm{m} \mu$ /day, but special devices are needed to this aim. The reason for the advantage of a slow speed can be deduced from the latter publication: when one electrode within an array of individually movable electrodes is advanced very slowly, the signals recorded at the other electrodes tend to remain unchanged. It follows that a faster speed induces a farther-reaching deformation of tissue or, expressed otherwise, mechanical tissue stress induced by electrode movement requires some time to decay. If no such time is available, the greater stress can be expected to engender a more important gliosis.

On the other hand, it is most likely a consequence of the inverted insertion method that the short-term stability of the neuronal signal was excellent within each experimental session although the animal was only partially restrained and the head was free to move in all directions. This is particularly noteworthy since rapid movements of the head, as well as the increase of intracranial pressure following movements or stretching of the animal, are known to produce artifacts or loss or changing of neuronal signal. Our result is particularly interesting since it allows to perform neurophysiological experiments collecting reliable data in more ecological contexts.

Lastly, some practical issues for monkey experiments will be considered. In principle the multiple plug carrying the headstage on the head of the animal can be held by a much smaller subcutaneous titanium construction (Krüger and Hauser, 2004), instead of the block of acrylic cement. The multiple plug on the head of the animal should neither have male nor female junctions. Rather, the contacts on the head should be made from noble metals and be flat or shallowly hollow, so that they can be easily cleaned, and no male contacts can break. The output cables of the headstage must support long-term manipulatory stress, or be easily accessible for repair. One would also have to test whether contacting materials such as the silver grain epoxy (see Manufacture of the electrode) transmit microvolt signals in an invariable way for many years. It is advisable to consider from the beginning that the personnel working for many years with such a preparation may change, and that it is crucial to maintain a very high level of technical care over many years. Lastly, we want to note that the individual wires can be seen in vivo in classical X-ray radiographs if they are made by heavy-atom metals such as platinum-iridium instead of the nickel-chromium alloy used here, and when a high-resolution film is used. Stereoscopic in vivo inspection of the electrodes is then possible.

On the other hand, the present technique requires some care about the selection of the path taken by the curved cannula because most lesions were observed there, and near the root of the brush of microwires. Possibly a method to introduce the brush much more slowly will give better results in this respect. At the moment, our technique was limited insofar as we only took into account the MRIbased knowledge of the target location; since the microwires are not visible during surgery, a great improvement could be obtained if the entire path taken by the electrodes and the cannula through the gray matter could be optimized with the aid of pre-operative MRI imagery.

There is an overall advice which is valid for most technical and scientific questions, as well as for dismissing objections of various kinds: increase the number of electrodes! The present technique easily allows this. It is cheap, the electrodes can be made in the laboratory, there are no additional contacts between electrode tips and cranial plug (for this point, see the report of Hochberg et al., 2006), the processing of data from hundreds of channels is no longer a problem, and fully valid experiments can be done, within $1 \mathrm{~h}$, from the very beginning of taking the animal from the keeping station until bringing it back.

\section{ACKNOWLEDGMENTS}

This work has been supported, in its initial part, by Deutsche Forschungsgemeinschaft (Kr 323-11/1 to Jürgen Krüger). Fausto Caruana was supported by EU Contract 027017 "Neuroprobe". We thank F. Botti, A. Bruzzo, B. Engel, B. Friedrich, V. Gallese, F. Grammont, I. Intskirveli, A. Jezzini, S. Lehmann, L. Milito, L. Nanetti, S. Rozzi for contributions in different periods of this project. 


\section{REFERENCES}

Andersen, R.A., Musallam, S., and Pesaran, B. (2004). Selecting the signals for a brain-machine interface. Curr. Opin. Neurobiol. 14, 720-726.

Dalla Volta, R., Grammont, F., Bruzzo, A., and Krüger, J. (2002). Chronical multielectrode recording applied to monkey ventral premotor cortex Poster presented at "The Human Brain.” 1st International conference, October 2002, Fondazione IRCCS Santa Lucia, Roma.

Donoghue, J. P. (2008). Bridging the brain to the world: a perspective on neural interface systems. Neuron 60 , 511-521.

Fraser, G. W., Chase, S. M., Whitford, A., and Schwartz, A. B. (2009). Control of a brain-computer interface without spike sorting. J. Neural Eng. 6, 055004 (8 pp).

Hatsopoulos, N. G., and Donoghue, J. P. (2009). The science of neural interface systems. Annu. Rev. Neurosci. 32, 249-266.

Hochberg, L. R., Serruya, M. D., Friehs, G. M., Mukand, J.A., Saleh, M., Caplan, A. H., Branner, A., Chen, D., Penn, R. D. and Donoghue, J. P. (2006). Neuronal ensemble control of prosthetic devices by a human with tetraplegia. Nature 442, 164-171.

Jain, N., Qi, H.-X., and Kaas, J. H. (2001). Long-term chronic multichannel recordings from sensorimotor cortex and thalamus of primates in advances in neural population coding. Prog. Brain Res. 130, 64-72.

Krüger, J., and Hauser, J. (2004). Evoking defined gaze directions in untrained awake monkeys. J. Neurosci. Methods 137, 185-192.

Laubach, M., Wessberg, J., and Nicolelis, M.A. (2000). Cortical ensemble activity increasingly predicts behaviour outcomes during learning of a motor task. Nature 405, 567-571.

Mehring, C., Rickert, J., Vaadia, E., de Oliveira, S. C., Aertsen, A., and Rotter, S. (2003). Inference of hand movements from local field potentials in monkey motor cortex. Nat. Neurosci. 6, 1253-1254.

Nicolelis, M. A. L., Dimitrov, D., Carmena, J. M., Crist, R., Lehew, G. Kralik, J. D., and Wise, S. P. (2003) Chronic, multisite, multielectrode recordings in macaque monkeys. Proc. Natl. Acad. Sci. USA 100, 11041-11046.

Nordhausen, C. T., Maynard, E. M. and Normann, R. A. (1996). Single unit recording capabilities of a 100 micorelectrode array. Brain Res. 726, 129-140.

Porada, I., Bondar, I., Spatz, W. B., and Krüger, J. (2000). Rabbit and monkey visual cortex: more than a year of recording with up to 64 microelectrodes. J. Neurosci. Methods 95, 13-28.
Rousche, P. J., and Normann, R.A. (1998) Chronic recording capability of the Utah intracortical electrode array in cat sensory cortex. J. Neurosci. Methods $82,1-15$.

Serruya, M. D., Hatsopoulos, N. G. Paninski, L., Fellows, M. R., and Donoghue, J. P. (2002). Instant neural control of a movement signal. Nature 416, 141-142.

Stark,E., and Abeles, M. (2007). Predicting movement from multiunit activity. $J$. Neurosci. 27, 8387-8394.

Szarowski, D. H., Andersen, M. D. Retterer, S., Spence, A. J., Isaacson M., Craighead, H. G., Turner, J. N., and Shain, W. (2003). Brain responses to machined silicon devices. Brain Res. 983, 23-35.

Tolias, A. S., Ecker, A. S., Siapas, A. S., Hoenselaar, A., Keliris, G. A., and Logothetis, N. K. (2007). Recording chronically from the same neurons in awake, behaving primates. $J$. Neurophysiol. 98, 3780-3790.

Umiltà, M. A., Escola, L., Intskirveli, I., Grammont, F., Rochat, M., Caruana F., Jezzini, A., Gallese, V., and Rizzolatti, G. (2008). How pliers become fingers in the monkey motor system. Proc Natl. Acad. Sci. USA 105, 2209-2213.

Velliste, M., Perel, S., Spalding, M. C. Whitford, A. S., and Schwartz, A. B. (2008). Cortical control of a prosthetic arm for self-feeding. Nature 453, 1098-1101.
Waldert, S., Pistohl, T., Braun, C., Ball, T., Aertsen, A., and Mehring, C. (2009). A review on directional information in neural signals for brain-machine interfaces. J. Physiol. Paris 103, 244-254.

Yamamoto, J., and Wilson, M. A. (2008). Large-scale chronically implantable precision motorized microdrive array for freely behaving animals. $J$. Neurophysiol. 100, 2430-2440.

Conflict of Interest Statement: The authors declare that the research was conducted in the absence of any commercial or financial relationships that could be construed as a potential conflict of interest.

Received: 02 January 2010; paper pending published: 14 February 2010; accepted: 30 April 2010; published online: 28 May 2010

Citation: Krüger J, Caruana F, Dalla Volto $R$ and Rizzolatti $G$ (2010) Seven years of recording from monkey cortex with a chronically implanted multiple microelectrode. Front. Neuroeng. 3:6. doi: 10.3389/ fneng.2010.00006

Copyright (C) 2010 Krüger, Caruana, Dalla Volta and Rizzolatti. This is an open-access article subject to an exclusive license agreement between the authors and the Frontiers Research Foundation, which permits unrestricted use, distribution, and reproduction in any medium, provided the original authors and source are credited. 